ARTICLE

Received 1 Oct 2015 | Accepted 22 Dec 2015 | Published 29 Jan 2016

DOI: $10.1038 /$ ncomms10510 OPEN

\title{
The Hippo signalling pathway maintains quiescence in Drosophila neural stem cells
}

Rouven Ding ${ }^{1}$, Kevin Weynans ${ }^{1, \dagger}$, Torsten Bossing ${ }^{2}$, Claudia S. Barros ${ }^{3} \&$ Christian Berger $^{1}$

Stem cells control their mitotic activity to decide whether to proliferate or to stay in quiescence. Drosophila neural stem cells (NSCs) are quiescent at early larval stages, when they are reactivated in response to metabolic changes. Here we report that cell-contact inhibition of growth through the canonical Hippo signalling pathway maintains NSC quiescence. Loss of the core kinases hippo or warts leads to premature nuclear localization of the transcriptional co-activator Yorkie and initiation of growth and proliferation in NSCs. Yorkie is necessary and sufficient for NSC reactivation, growth and proliferation. The Hippo pathway activity is modulated via inter-cellular transmembrane proteins Crumbs and Echinoid that are both expressed in a nutrient-dependent way in niche glial cells and NSCs. Loss of crumbs or echinoid in the niche only is sufficient to reactivate NSCs. Finally, we provide evidence that the Hippo pathway activity discriminates quiescent from non-quiescent NSCs in the Drosophila nervous system.

\footnotetext{
${ }^{1}$ Institute of Genetics, Johannes Gutenberg University, 55099 Mainz, Germany. ${ }^{2}$ School of Biomedical and Healthcare Sciences, Plymouth University, PL4 8AA Plymouth, UK. ${ }^{3}$ Peninsula School of Medicine, Plymouth University, PL6 8BU Plymouth, UK. † Present address: Institute of Reconstructive Neurobiology, University Bonn, 53127 Bonn, Germany. Correspondence and requests for materials should be addressed to C.B.

(email: bergerc@uni-mainz.de).
} 
S tem cells are undifferentiated cells that have the unique ability to produce differentiating daughter cells and retain their identity by a process called self-renewal. Stem cells can exhibit a remarkable proliferative capacity, for example, during development or regenerative processes ${ }^{1,2}$. Deregulation of stem cell proliferation can lead to tumour formation or to a premature depletion of the progenitor pool $^{3}$. Thus, stem cell proliferation has to be tightly regulated according to the cellular or organismal context. When proliferation is not required, stem cells are maintained in a state of quiescence in the G0-phase and need to be activated by systemic or local signals ${ }^{3,4}$. In Drosophila, neural stem cells (NSCs) proliferate in two phases ${ }^{5}$. The embryonic phase generates all cells of a functional larval central nervous system (CNS), while in the second proliferative phase cells forming the adult CNS are produced. In late embryonic stages NSCs enter quiescence, which requires intrinsic transcription factors ${ }^{6,7}$.

Changes in the physiological condition of the animal in response to feeding at early larval stages causes reactivation of $\mathrm{NSCs}^{8}$. The amino-acid sensing fat body releases systemic signals in response to the increase in dietary amino acids ${ }^{8,9}$ and CNS glial cells translate these signals into a local activating signal. They produce and secrete insulin-like peptides that activate the insulin/ insulin-like growth factor signalling pathway in $\mathrm{NSCs}^{10,11}$. An initial step during reactivation is the drastic increase in NSC cell size from $4-5 \mu \mathrm{m}$ during quiescence to $10-15 \mu \mathrm{m}$ depending on the type of $\mathrm{NSC}^{5,12}$. Thus, growth in preparation for cell division is one of the initial hallmarks of NSC reactivation. The mechanisms regulating quiescence are less well understood. Glial cells secrete a glycoprotein (anachronism) that keeps NSCs in quiescence, but the precise molecular mechanism remains unknown ${ }^{13}$.

One of the major pathways that controls organ growth and cell proliferation in Drosophila and vertebrates is the conserved Salvador/Hippo/Warts signalling pathway $(\mathrm{SHW})^{14-16}$. The SHW consists of a growth-repressive kinase cascade that modulates the activity of the transcriptional co-activator Yorkie (YAP/TAZ in vertebrates). The Hippo kinase activates the Warts kinase, which in turn directly phosphorylates Yorkie, creating a 14-3-3 binding site that restricts nuclear import and inactivates Yorkie $^{17,18}$. If Hippo/Warts are inactive, non-phosphorylated Yorkie enters the nucleus and binds to transcription factors like Scalloped ${ }^{19,20}$ and activates its transcriptional program promoting cell growth and proliferation ${ }^{21,22}$. Numerous upstream regulators of the SHW have been identified, including cell-cell contact, the actin cytoskeleton, G-protein coupled receptors or planar and apico-basal cell polarity ${ }^{23}$.

In the vertebrate skin or the liver, de-repression of YAP has been shown to promote stem cell proliferation ${ }^{24}$. However, whether this is true in NSCs and whether changes in Yorkie/YAP activity are causative for altering growth and proliferation during normal CNS development remains unclear. In Drosophila, the SHW has been implicated in CNS development of the neuroepithelium and of glial cells in the optic lobe, and in cell growth of a specific population of glial cells (subperineural glial cells $^{25-27}$. However, for central brain NSCs, no function has been attributed to the SHW.

Here, we show that the SHW maintains quiescence of NSCs at the transition from embryo to larval life in Drosophila. Loss of the core kinases hippo/warts, or upstream regulators kibra/Merlin/ expanded, leads to a premature initiation of cell growth and proliferation and thus reactivation from quiescence. Yorkie is inactive in quiescent NSCs and is necessary and sufficient for the reactivation and proliferation of NSCs. Cell-cell contact proteins Crumbs and Echinoid are expressed in both glial cells and NSCs and regulate the activity of Hippo and Warts, possibly via homophilic interactions in trans. The expression of Crumbs and Echinoid in glial cells and NSCs is nutrition-dependent, and their premature loss in glial cells is sufficient to initiate reactivation of NSCs. Moreover, the Yorkie activity discriminates between quiescent and non-quiescent NSCs, placing the SHW as a major regulator of growth in cellular quiescence in Drosophila NSCs.

\section{Results}

Loss of Hippo signalling causes premature NSCs reactivation. To identify novel regulators of quiescence in NSCs, we depleted known growth regulators using RNAi-mediated gene knockdown in the insc-GAL4 pattern ${ }^{28}$. We scored NSCs (Deadpan-positive cells) cell size and proliferation rate (phosphohistone $\mathrm{H} 3(\mathrm{pH} 3)$ positive NSCs) $4 \mathrm{~h}$ after larval hatching (ALH), when all NSCs are quiescent-small in cell size $(\sim 4-5 \mu \mathrm{m})$ and non-proliferative (Fig. 1a,g,h) $5,10,12$. Exceptions are four NSCs of the mushroom body (MBNBs) and one ventrolateral NSC (INSC) that do not enter quiescence, have a large cell diameter and constantly proliferate (Fig. 1a) ${ }^{29}$. These 'non-quiescent NSCs' are quantified independently from all quiescent NSCs. First, we describe the fate of quiescent NSCs.

Knockdown of the core kinases of the SHW hippo or warts induces a marked premature increase in NSC cell size (Fig. 1b,c) from $4.5 \mu \mathrm{m}$ (median, maximum $6.5 \mu \mathrm{m}$ ) in control brains $4 \mathrm{~h}$ ALH to $7 \mu \mathrm{m}$ (median, maximum $13 \mu \mathrm{m}$; Fig. 1g). Since this suggests an early exit from quiescence, we tested for entry into S-phase using antibody staining for the S-phase cyclin CycE. We observed an increase in CycE-positive NSCs upon warts-RNAi (Supplementary Fig. 1a,b). Consequently, the number of pH3-positive mitotic NSCs was also significantly increased (Fig. 1h). Next, we examined the known upstream regulators of the SHW expanded, kibra or Merlin for their function in NSCs. Indeed, RNAi showed similar albeit less-pronounced effects and caused premature cell growth at $4 \mathrm{~h}$ ALH (Fig. 1d-g).

To ensure that this phenotype is not because of an impaired entry into quiescence, we analysed trans-heterozygous $h p o^{J M 1} / h p o^{K C 202}$ mutants ${ }^{30,31}$ at hatching $(0-2 \mathrm{~h} \mathrm{ALH})$ and $4 \mathrm{~h}$ ALH (Supplementary Fig. 1c,d), and stage-17 embryonic brains of $w t s$-RNAi (Supplementary Fig. 1e,f). In both situations NSCs did not show increased cell sizes or mitotic activity revealing a normal phase of quiescence (stage-17 at $0-2 \mathrm{~h} \mathrm{ALH)}$ ). Interestingly, $h p o$ mutant larvae exhibited a mild but significant increase in cell size at $4 \mathrm{~h}$ ALH mimicking the reactivation phenotype in wts-RNAi (Fig. 1i). Finally, we used the temperature-sensitive GAL4 repressor system GAL80ts to restrict the RNAi expression to only larval stages. Indeed, upon larval stage-restricted $w t s$ - or hpo-RNAi in NSCs we could monitor a similar increase in NSC diameter at $4 \mathrm{~h} \mathrm{ALH} \mathrm{(Fig.} \mathrm{1j).}$

Thus, the SHW acts in Drosophila NSCs to maintain quiescence and cell-autonomous loss of pathway components leads to premature exit from quiescence.

Yorkie relocates to the nucleus during reactivation. If the SHW maintains quiescence, the main effector Yorkie $^{32}$ should be inactive and excluded from the nucleus in quiescent NSCs ${ }^{17,18}$, whereas we should observe nuclear localization in reactivated NSCs (24h ALH). Antibody staining revealed no nuclear localization of Yorkie in quiescent NSCs (Fig. 2a,d and Supplementary Fig. 2). In contrast, at $24 \mathrm{~h} \mathrm{ALH} \mathrm{a} \mathrm{clear} \mathrm{nuclear}$ localization of Yorkie in reactivated NSCs can be detected (Fig. 2b,d and Supplementary Fig. 2). Since wts-RNAi caused premature reactivation $4 \mathrm{~h} \mathrm{ALH}$, we tested for premature nuclear localization of Yorkie and could monitor an increase in Yorkie protein levels and nuclear localization in NSCs that display a clear increase in cell diameter (Fig. 2c). Moreover, since 

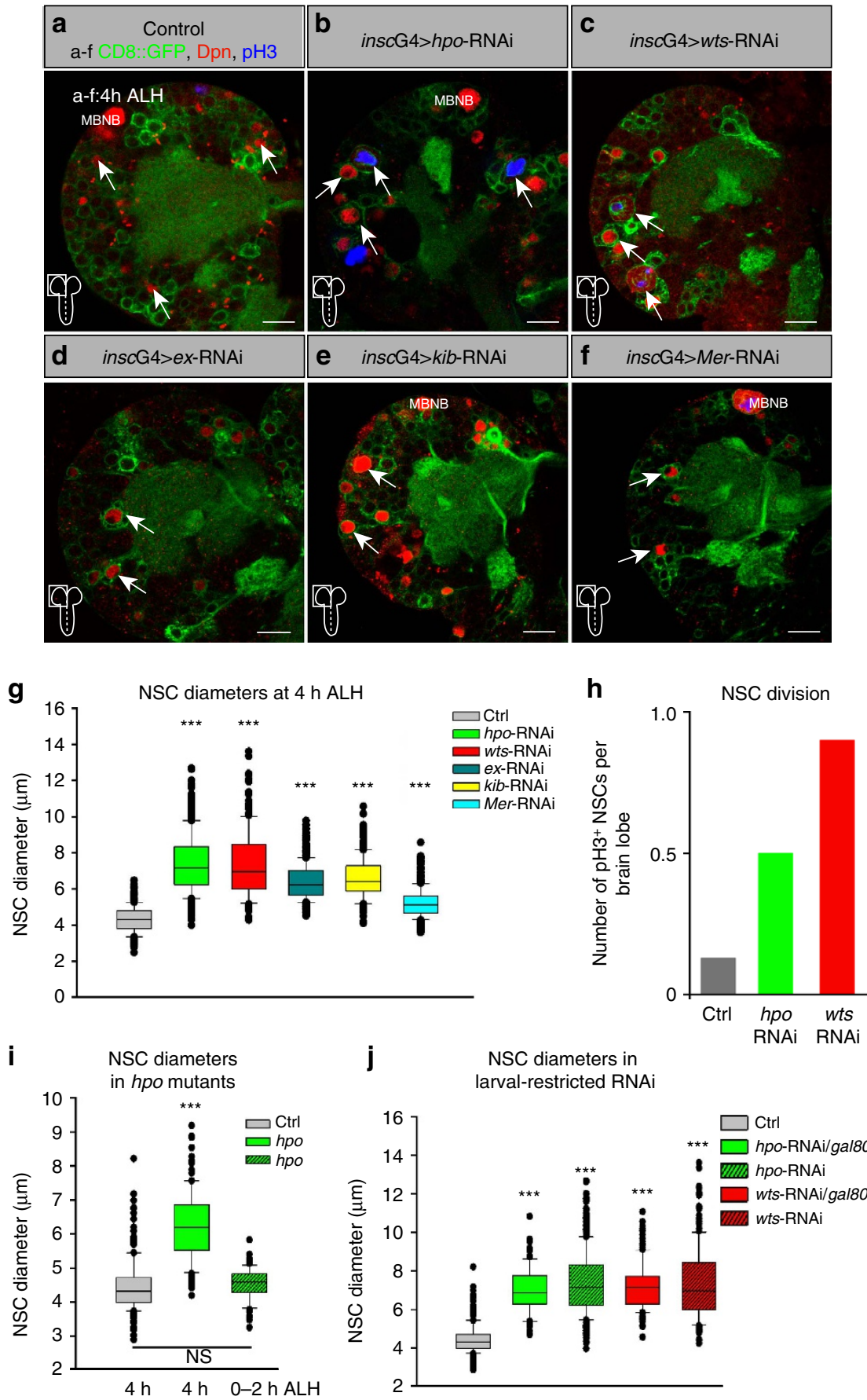

j NSC diameters in larval-restricted RNA

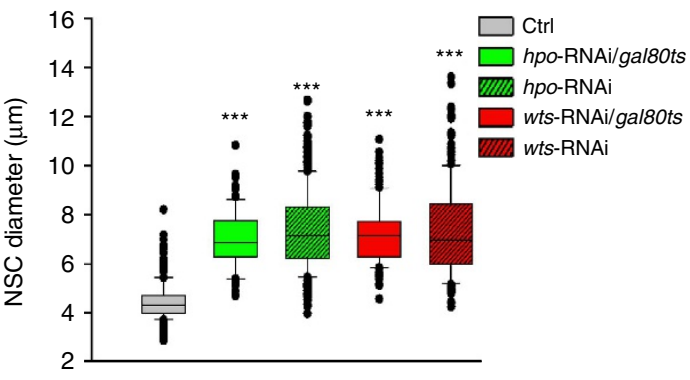

Figure 1 | The SHW signalling pathway maintains quiescence in larval NSCs. (a) Wild-type larval brain lobe (inscG4 > UAS-CD8::GFP, Control) 4 h ALH. Quiescent NSCs are small in cell size. NSCs of the mushroom body (MBNB) are big in cell size. No pH3-positive NSCs can be observed. (b-f) NSC-specific RNAi (inscG4 > UAS-CD8::GFP and respective construct) of hippo (hpo)(b), warts (wts)(c), expanded (ex) (d), kibra (kib) (e) or Merlin (Mer) (f) leads to premature cell growth and cell division (observed in hpo and wts-RNAi) of NSCs. Arrows depict examples of NSCs. (g,h) Quantification of NSC cell diameters $(\mathbf{g})$ and proliferation $(\mathbf{h})$ in RNAi for Hippo pathway components. $(\mathbf{g}){ }^{\star \star \star} P<0.001$. Wilcoxon rank sum test. Median and s.d. were calculated from two biological replicates. Control $n=355$ NSCs (7 brain lobes); inscG4 $>$ hpo-RNAi, $n=575$ NSCs (11 brain lobes); inscG4 $>$ wts-RNAi, $n=236$ NSCs ( 5 brain lobes); inscG4 > expanded-RNAi, $n=400$ NSCs ( 8 brain lobes); inscG4 > kibra-RNAi, $n=266$ NSCs ( 5 brain lobes); inscG4 > Mer-RNAi, $n=506$ NSCs (10 brain lobes); all $4 \mathrm{~h} \mathrm{ALH.} \mathrm{(h)} \mathrm{Number} \mathrm{of} \mathrm{NSCs} \mathrm{in} \mathrm{mitosis} \mathrm{(pH3-positive)} \mathrm{at} 4 \mathrm{~h} \mathrm{ALH}$ per brain lobe in wild-type (7 brain lobes) and hpo- and wtsRNAi (11 or 5 brain lobes). (i) Quantification of NSC cell diameters in $h p o^{J M 1} / h_{p o} K C 202$ (hpo in figure) trans-heterozygous mutants at $0-2 \mathrm{~h}$ ALH and $4 \mathrm{~h}$ ALH. ${ }^{\star * \star} P<0.001$. Wilcoxon rank sum test. Median and s.d. were calculated from two biological replicates. Control $4 \mathrm{~h}$ ALH, $n=150 \mathrm{NSCs}$ ( 3 brain lobes);

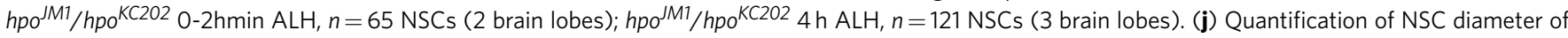
larval-restricted RNAi of hpo ( $n=120$ NSCs in 8 brain lobes) and wts ( $n=172$ NSCs in 10 brain lobes) in NSCs using the GAL80ts system. For comparison hpo- and wts-RNAi measurements without GAL80ts are added. All images are single focal planes, anterior up; Scale bar, $10 \mu \mathrm{m}$. NSC in red (Deadpan), CD8::GFP in green and phosphohistone $\mathrm{H} 3$ in blue (pH3). Pictograms denote the area of the brain shown in the picture. See also Supplementary Fig. 1. 


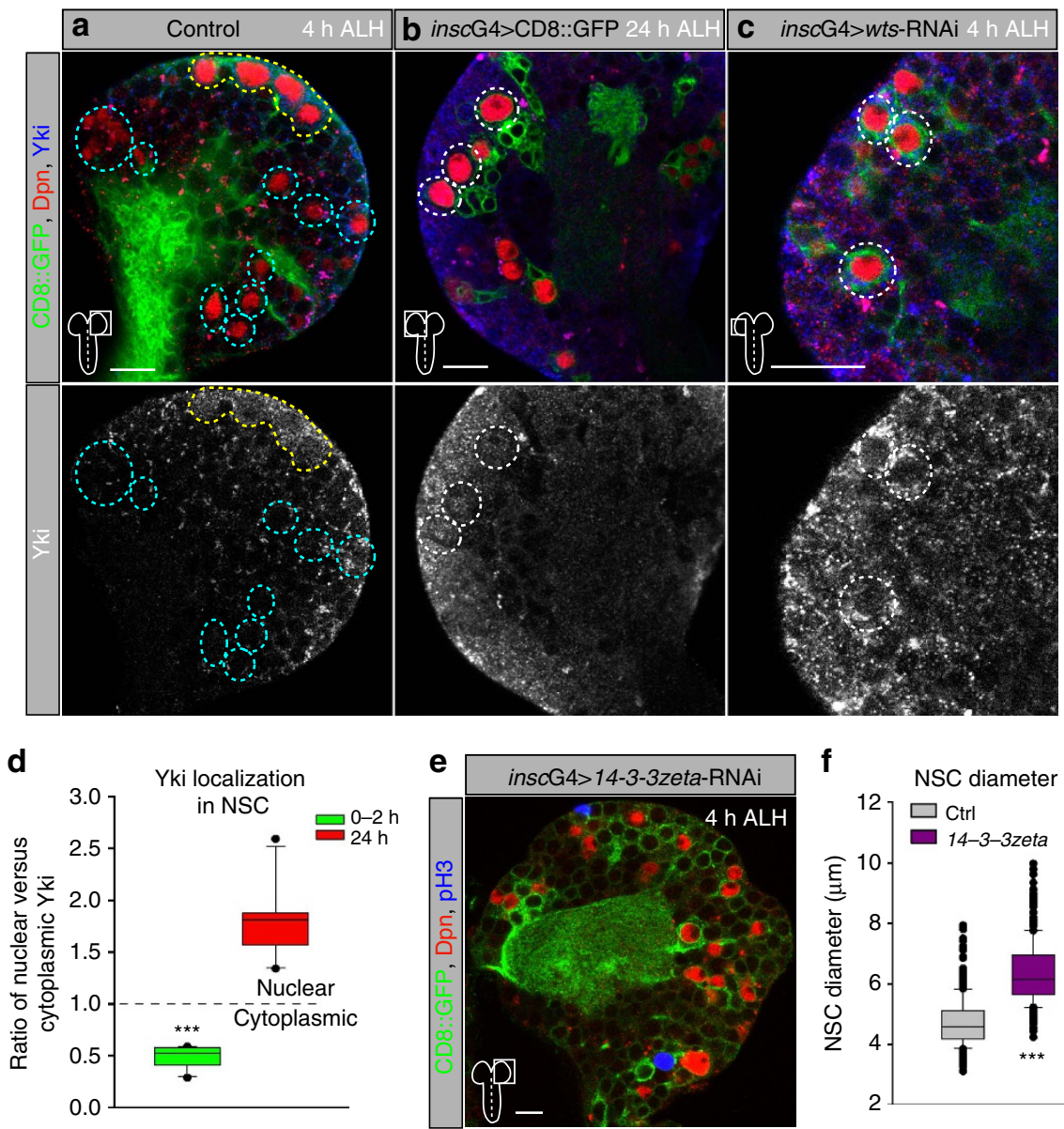

Figure 2 | Yorkie is cytoplasmic during quiescence and re-localizes to the nucleus during reactivation of NSCs. (a-c) Subcellular localization of Yorkie in quiescent NSC at $4 \mathrm{~h} \mathrm{ALH} \mathrm{(a,} \mathrm{cyan} \mathrm{circles,} \mathrm{yellow} \mathrm{circles} \mathrm{show} \mathrm{non-quiescent} \mathrm{MBNBs),} \mathrm{in} \mathrm{reactivated} \mathrm{NSCs} \mathrm{at} 24 \mathrm{~h}$ ALH (b, white circles) and in prematurely reactivated NSCs of wts-RNAi at $4 \mathrm{~h} \mathrm{ALH} \mathrm{(c,} \mathrm{white} \mathrm{circles).} \mathrm{Lower} \mathrm{panels} \mathrm{show} \mathrm{only} \mathrm{the} \mathrm{Yorkie} \mathrm{channel} \mathrm{in} \mathrm{monochrome.} \mathrm{Circles} \mathrm{depict}$ examples of NSCs. (d) Quantification of the nuclear versus cytoplasmic localization of Yorkie in NSCs at 0-2 h (green, $n=20,5$ brains from different animals) and $24 \mathrm{~h}$ (red, $n=20,5$ brains from different animals) ALH. ${ }^{* \star \star} P<0.001$. Wilcoxon rank sum test. (e) NSC-specific RNAi of $14-3-3 z e t a$ leads to premature growth of NSCs at $4 \mathrm{~h} \mathrm{ALH}$. (f) Quantification of NSC cell diameters in wild-type and 14-3-3zeta-RNAi at $4 \mathrm{~h} \mathrm{ALH}$. ${ }^{\star \star \star} P<0.001$. Wilcoxon rank sum test. Median and s.d. were calculated from two biological replicates. Control $n=347$ NSCs ( 7 brain lobes); inscG4 $>14-3-3 z e t a-R N A i, n=250$ NSCs ( 5 brain lobes). All images are single focal planes, anterior up. Scale bar, $10 \mu \mathrm{m}$. See also Supplementary Fig. 2.

phosphorylated Yorkie binds to $14-3-3$ and stays inactive, we tested the loss of 14-3-3-zeta with RNAi and observed premature growth of NSCs at $4 \mathrm{~h} \mathrm{ALH} \mathrm{(Fig.} \mathrm{2e,f),} \mathrm{presumably} \mathrm{owing} \mathrm{to} \mathrm{early}$ activity of Yorkie. Thus, Yorkie is inactive in NSCs during quiescence, and is activated and localizes to the nucleus during reactivation or upon $w t s$-RNAi.

Yorkie is necessary and sufficient for growth and proliferation. To determine whether Yorkie is also necessary for NSC growth and proliferation, we analysed $y k i^{B 5}$ null mutants ${ }^{32}$. Homozygous $y k i^{B 5}$ mutants are embryonically semi-lethal and most larvae die at approximately $48 \mathrm{~h} \mathrm{ALH}$. Whereas, wild-type NSCs at $48 \mathrm{~h}$ $\mathrm{ALH}$ have been reactivated and are highly proliferative (Fig. 3a,c,d), no reactivation of quiescent NSCs can be observed in the $y k i^{B 5}$ mutants (no cell growth and no pH3-positive NSCs; Fig. 3b-d). Moreover, NSCs cell size and their mitotic index at $48 \mathrm{~h}$ ALH revealed that $y k i^{B 5}$-mutant NSCs resemble quiescent NSCs (Fig. 3c,d). Next, we tested whether early expression of a constitutively active form of Yorkie (UAS- $\left.y k i^{S 168 A}\right)^{17}$ is sufficient to reactivate NSCs. Indeed, at $4 \mathrm{~h}$ ALH we observed a significant increase in NSCs cell diameter, which was also present when restricting the expression to only larval stages using the GAL80ts system (Fig. 3e,f). We conclude that Yorkie function is necessary and sufficient for NSCs reactivation and initiation of growth and proliferation.

Yorkie activates the bantam microRNA during reactivation. Next we tested if the expression of the Yorkie target genes four-jointed $(f j-l a c Z)^{33}$, expanded ${ }^{34}$ and the microRNA bantam ${ }^{35}$ correlates with the subcellular translocation of Yorkie during reactivation. Fj-lacZ and expanded showed only weak expression in quiescent NSCs (4h ALH) but clear upregulation at $48 \mathrm{~h} \mathrm{ALH}$ (Supplementary Fig. 3a,b). We analysed the expression and activity of bantam that is known to regulate proliferation and growth $^{35-38}$ by using a GFP-sensor system ${ }^{36}$. The loss of GFP expression and thus the activity of bantam coincides with the activation of Yorkie, as quiescent NSCs (4h ALH) show strong GFP staining (Fig. 4a, upper panels) and reactivated NSCs (24 h ALH) have markedly reduced GFP signals monitoring bantam activity (Fig. 4a, lower panels). We combined the bantam sensor with wts-RNAi and examined an early activity of bantam at $4 \mathrm{~h} \mathrm{ALH} \mathrm{(Fig.} \mathrm{4b),} \mathrm{suggesting} \mathrm{that} \mathrm{premature} \mathrm{reactivation} \mathrm{by}$ 

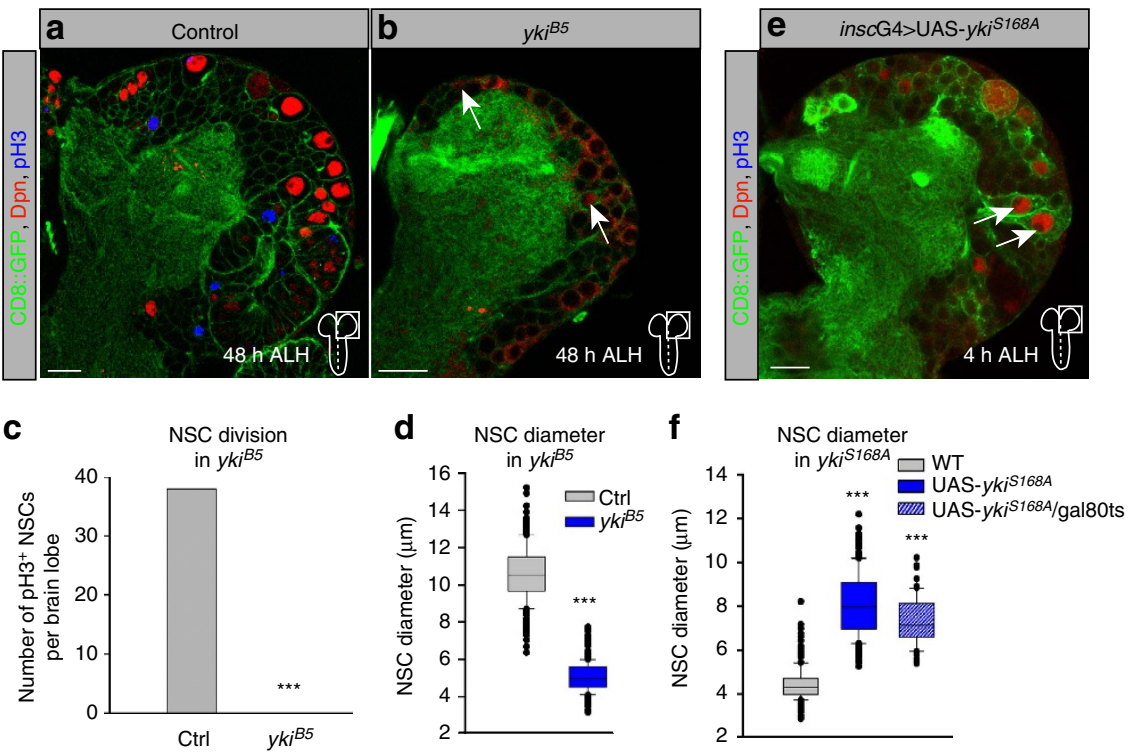

Figure 3 | Yorkie is necessary and sufficient for NSCs growth and proliferation. (a,b) NSC growth and proliferation is impaired in $y k i^{B 5}$ deletion mutants (b) compared with wild-type NSCs at $48 \mathrm{~h} \mathrm{ALH} \mathrm{(a).} \mathrm{(c)} \mathrm{Average} \mathrm{number} \mathrm{of} \mathrm{NSCs} \mathrm{in} \mathrm{mitosis} \mathrm{(pH3-positive)} \mathrm{at} 48 \mathrm{~h} \mathrm{ALH}$ in wild-type and $y k^{B 5}$ deletion mutants. (d) Quantification of NSC cell diameters $y k^{B 5}$ deletion mutants at $48 \mathrm{~h}$ ALH. ${ }^{\star \star \star} P<0.001$. Wilcoxon rank sum test. Median and s.d. were calculated from two biological replicates. Control $n=240$ NSCs ( 5 brain lobes); $y k i{ }^{B 5}, n=459$ NSCs (10 brain lobes). (e) Expression of $y k i S 168 A$ in NSCs is sufficient to reactivate NSCs $4 \mathrm{~h} \mathrm{ALH}$. (f) Quantification of NSC cell diameters in ectopic expression of inscG4 $>$ ykiS168A (blue) or larval-restricted expression using the GAL80ts system (blue with white lines) at $4 \mathrm{~h} \mathrm{ALH.}{ }^{\star \star \star} P<0.001$. Wilcoxon rank sum test. Median and s.d. were calculated from two biological replicates. Control $n=150$ NSCs (3 brain lobes); inscG $4>$ ykiS168A, $n=200$ NSCs ( 4 brain lobes); inscG4 > ykiS168A; tubPGAL80ts, $n=62$ NSCs (4 brain lobes). All images are single focal planes, anterior up. Scale bar, $10 \mu \mathrm{m}$.

$w t s$-RNAi expression causes early activation of the Yorkie downstream target bantam. To test if bantam is also necessary for NSC reactivation, we analysed bantam $\Delta 1$ deletion mutants at $24 \mathrm{~h}$ ALH and observed NSC reactivation in the brain, but markedly reduced cell size and proliferative capacity (Fig. 4c-f). This effect seemed stronger in NSCs of the ventral nerve cord (VNC) at $24 \mathrm{~h} \mathrm{ALH,} \mathrm{which} \mathrm{in} \mathrm{bantam} 11$ mutants were indistinguishable from quiescent NSCs in control VNCs at $4 \mathrm{~h}$ $\mathrm{ALH}$, with severely reduced cell sizes and nearly no $\mathrm{pH} 3$-positive NSCs (Fig. 4e,f). The difference between brain and VNC NSCs can be attributed to the spatiotemporal progression of NSC reactivation from anterior to posterior, which is also reflected in the size distribution of the wild-type control (Fig. 4e). Thus, we conclude that bantam is an important target of Yorkie during reactivation of NSCs, yet other unknown targets are likely involved in growth and proliferation of NSCs.

SHW regulates reactivation depending on nutritional status. Because reactivation of NSCs is dependent on a nutritional stimulus and insulin signalling from CNS glia $8,10,11$, we tested if premature reactivation upon $w t s$-RNAi depends on nutrition. Gene knockdown of $w t$ in starved larvae resulted in a minor but still significant increase in cell size compared with $w t s$ knockdown in well-fed larvae (Supplementary Fig. 3c,d and Fig. 1c,g) but we could not detect pH3-positive NSCs. Thus, the SHW might regulate growth initiation of NSCs in parallel to the nutritional response model. This shows, that sensing of new nutritional resources occurs within the first $4 \mathrm{~h} \mathrm{ALH}$ and SHW can initiate cell growth, but reactivation even in loss of the SHW depends on the nutritional status of the organism.

Crumbs and Echinoid activate SHW during NSC quiescence. We sought to investigate how external signals regulated the cell-intrinsic, growth-repressing activity of the SHW during reactivation. Since a number of inter- and extracellular SHW regulators are known, we tested the transmembrane proteins Crumbs $^{39-42}$ and Echinoid ${ }^{43,44}$ for their role in NSCs quiescence. When targeting $c r b$ or ed by RNAi in NSCs, a significant increase in NSCs cell size can be measured (Fig. 5a and Supplementary Fig. $4 \mathrm{a}, \mathrm{b})$ and in response to crb/ed-RNAi reactivated NSCs showed nuclear localization of Yorkie (Fig. 5b). For ed we analysed a embryonic/larval non-lethal hypomorphic allele $\left(e d^{F 72}\right)$ that also exhibited premature reactivation revealed by increased NSC cell diameters (Fig. 5a) and incorporation of EdU monitoring S-phases (Supplementary Fig. 4c).

Since NSC reactivation involves niche glia cells ${ }^{10,11}$, we explored the role of niche signalling during quiescence. Using repo-GAL4 for glial-RNAi, we targeted $c r b$ or ed in glial cells only, which was sufficient to observe a similar significant but lesspronounced increase in NSC cell size compared with knockdown of $c r b$ or $e d$ in NSCs (Fig. 5c and Supplementary Fig. 4d,e). To exclude that this is a consequence of altered SHW in glial cells, we analysed NSCs behaviour in glial wts-RNAi. In contrast to $c r b$ - or ed-RNAi we could observe a premature growth initiation in subperineural glial cells as described before (Supplementary Fig. $4 \mathrm{f})^{27}$. The effect on reactivation of NSCs was stronger compared with glial $c r b$ or ed RNAi (Supplementary Fig. 4g). To test whether wts-RNAi and therefore premature glial growth can bypass the nutritional stimulus, we analysed the effect of nutritional deprivation. Similar to $w t s-\mathrm{RNAi}$ in NSCs in nutrition-deprived conditions, wts-RNAi in glial cells in starved larvae leads to a minor reactivation phenotype and a less pronounced but still significant increase in NSCs cell diameter (Supplementary Fig. 4g). Thus, premature glial growth in $w t s$-RNAi can initiate growth in NSCs but full reactivation is dependent on nutrition. Knockdown of $c r b$ or ed in glial cells did not result in a premature growth initiation in glial cells (compare Supplementary Fig. 4d,e with f). To show that the premature growth initiation in NSCs upon glial-RNAi of $c r b$ and/or $e d$ is 

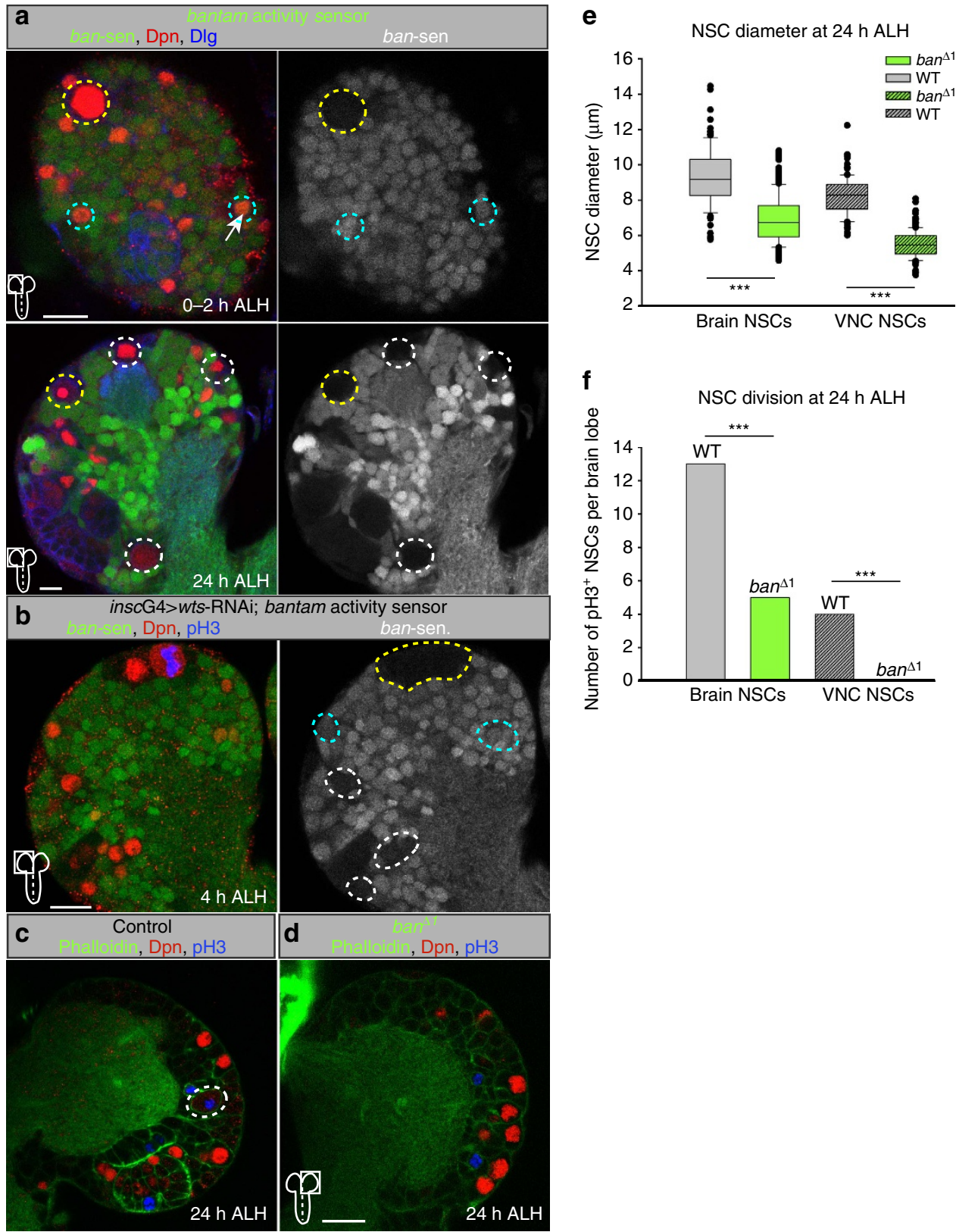

Figure 4 | The microRNA bantam is active during reactivation and necessary for NSC growth and proliferation. (a) bantam (ban)-activity sensor in brain lobes at 0-2 h (upper panels) and $24 \mathrm{~h}$ (lower panels) ALH. No GFP signal monitors ban activity. Quiescent NSCs (cyan circles) do not have active bantam (GFP-positive), whereas reactivated NSCs (white circles) or the MBNB (yellow circles) have active ban. Right panels show GFP channel in monochrome. (b) NSC-specific wts-RNAi leads to premature ban activity (loss of GFP) at $4 \mathrm{~h} \mathrm{ALH}$. (c,d) NSC growth and proliferation is decreased in ban ${ }^{1}$ mutants (d) compared with control (c, white circles marks NSC in division) at $24 \mathrm{~h} \mathrm{ALH}$. Phalloidin in green. (e,f) Quantification of NSC cell diameters (e) and proliferation (f) in ban $\Delta^{7}$ mutants at $24 \mathrm{~h} \mathrm{ALH}$. (e) ${ }^{* \star *} P<0.001$. Wilcoxon rank sum test. Median and s.d. were calculated from two biological replicates. Control brain $n=109$ NSCs (3 brain lobes); ban $\Delta^{1}$ brain $n=319$ NSCs (7 brain lobes); control VNC $n=109$ NSCs (3 VNC); ban $\Delta^{1}$ VNC $n=253$ NSCs ( 4 VNC). (f) Average number of NSCs in mitosis (pH3-positive) at $24 \mathrm{~h} \mathrm{ALH}$ in wild-type of 3 brain lobes and 3 VNCs and ban $\Delta^{1} 7$ brain lobes and 4 VNCs. All images are single focal planes, anterior up. Scale bar, $10 \mu \mathrm{m}$. See also Supplementary Fig. 3.

through altering the SHW pathway in NSCs, we made use of the bantam activity sensor. Indeed, we could monitor a premature activation of bantam (loss of GFP) in NSCs upon glial crb/ed RNAi (Fig. 5d). Therefore, knockdown of $c r b$ or ed in niche glial cells leads to premature reactivation of NSCs through altering Hippo activity in trans.

To test potential homophilic interactions of $\mathrm{Crumbs}^{45}$, we targeted crb simultaneously in both NSCs and glial cells by RNAi (insc-GAL4/ repo-GAL4) and observed an increase in the strength of premature reactivation of NSCs compared with glial knockdown alone (Fig. 5c,e and Supplementary Fig. 4h,i). Since the phenotype was similar to the NSC-specific RNAi of $c r b$ or ed alone we conclude that $c r b$ and ed act both in trans and in cis. Knockdown in glial cells removes the interaction in trans (between glial cells and NSCs) but leaves the interaction in cis (on NSCs), which causes a less-pronounced phenotype. Conversely, knockdown of $\mathrm{crb}$ or $e d$ in NSC or in NSC and glial cells simultaneously interrupts both interactions in cis and in trans and causes stronger phenotypes. 

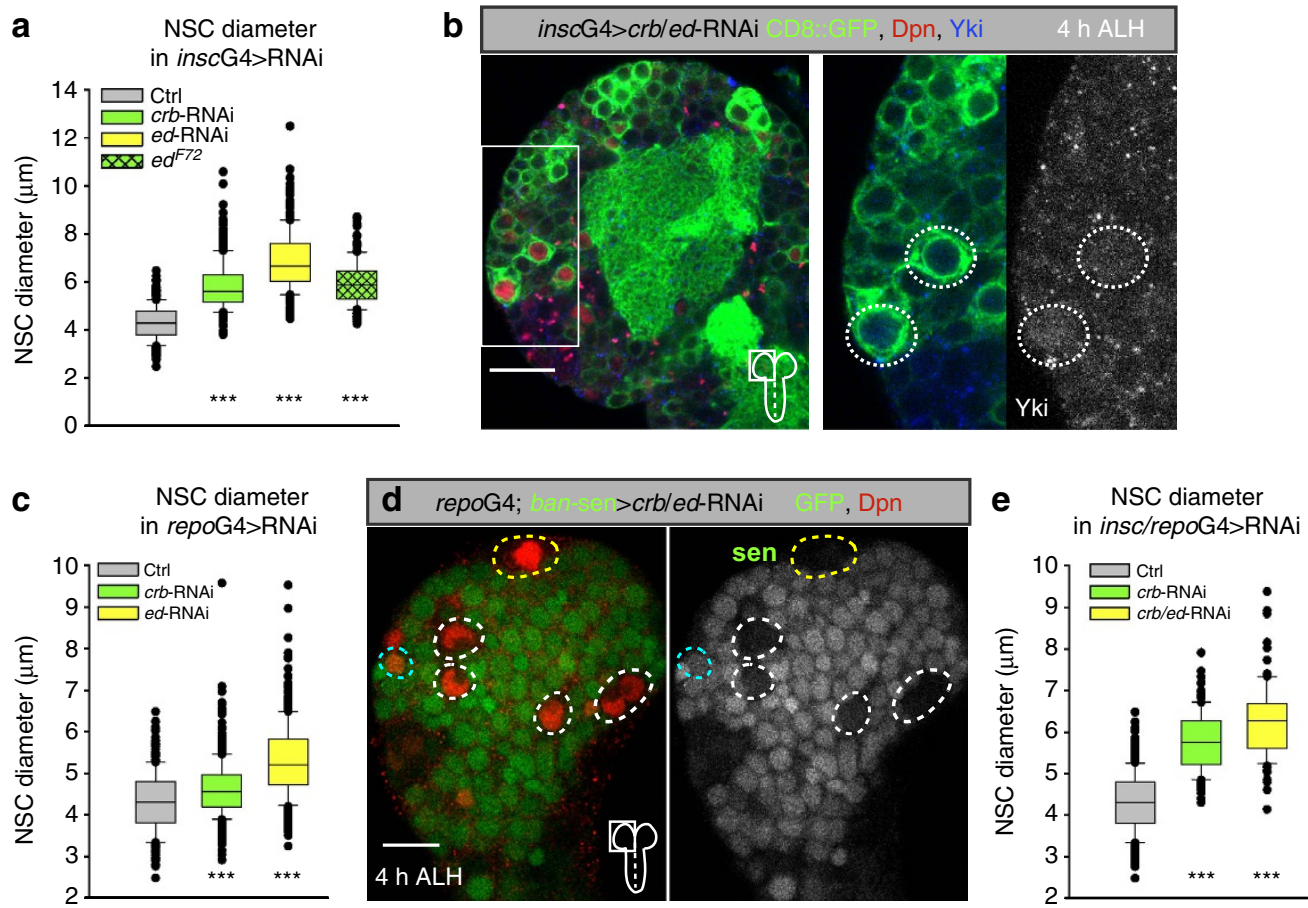

Figure 5 | Crumbs and Echinoid are required in NSCs and glial cells in cis and in trans to activate SHW during quiescence. (a) Quantification of NSC cell diameters in NSC-specific RNAi (inscG4) of $c r b$ or ed and in the ed ${ }^{F 72}$ mutant at $4 \mathrm{~h} \mathrm{ALH}$. ${ }^{\star \star \star} P<0.001$. Wilcoxon rank sum test. Median and s.d. were calculated from two biological replicates. Control brain $n=355$ NSCs (7 brain lobes); inscG4 >crb-RNAi, $n=313$ NSCs (7 brain lobes); inscG4 > ed-RNAi, $n=300$ NSCs ( 6 brain lobes); ed ${ }^{F 72} n=1,169$ (20 brain lobes). (b) NSC-specific crb/ed RNAi leads to premature nuclear Yorkie at $4 \mathrm{~h}$ ALH. Right panel shows a magnification of the marked area in the left panel. In the right panel only Yorkie (blue) and GFP (green) or Yorkie alone (monochrome) are shown. (c) Quantification of NSC cell diameters in Glia-specific RNAi (repoG4) of crb or ed at $4 \mathrm{~h} \mathrm{ALH.}{ }^{\star \star \star} P<0.001$. Wilcoxon rank sum test. Median and s.d. were calculated from two biological replicates. Control brain $n=255$ NSCs ( 5 brain lobes); repoG $4>c r b-R N A i, n=458$ NSCs ( 9 brain lobes); repoG4 $>$ ed-RNAi, $n=388$ NSCs (7 brain lobes). (d) Glial-specific crb/ed RNAi leads to premature bantam activity (loss of GFP) in NSCs (red, Deadpan) at $4 \mathrm{~h}$ ALH. Right panel shows GFP (ban-sensor, monochrome) signal alone. (e) Quantification of NSC cell diameters in Glia- and NSC-specific RNAi (inscG4; repoG4) of crb or Crumbs/ed at $4 \mathrm{~h} \mathrm{ALH} .{ }^{\star \star \star} P<0.001$. Wilcoxon rank sum test. Median and s.d. were calculated from two biological replicates. Control brain $n=145 \mathrm{NSCs}$ (3 brain lobes); inscG4; repoG4 >crb-RNAi, $n=125$ NSCs (3 brain lobes); inscG4; repoG4 >crb/ed-RNAi, $n=84$ NSCs (2 brain lobes). All images are single focal planes, anterior up. Scale bar, $10 \mu \mathrm{m}$. See also Supplementary Fig. 4.

Simultaneous glial- and NSC-specific knockdown of $c r b$ caused Yorkie nuclear localization in reactivated NSCs (Supplementary Fig. 4h) connecting crb function to the SHW. Finally, we assessed redundancy and performed double RNAi gene knockdowns of $c r b$ and ed simultaneously with the double driver GAL4 line (NSCs and glial cells concurrently), which caused NSC reactivation in the same degree as knockdown of ed alone (Fig. 5c,e and Supplementary Fig. 4i). Thus, other factors might compensate or influence the Hippo activity in NSCs along with $c r b$ or ed.

In conclusion, cell-contact inhibition of growth by niche glial cells through the SHW maintains quiescence in Drosophila NSCs. Interestingly, loss of Hippo signalling in the niche alone is able to initiate cell growth in NSC even during starvation.

Glial Crumbs and Echinoid expression depends on nutrition. Both Crumbs ${ }^{46}$ and Echinoid ${ }^{47}$ are expressed in epithelial cells and their role in glial cells and NSCs was surprising. Using a functional Crumbs::GFP fusion protein ${ }^{48}, c r b$-mRNA in situ (Supplementary Fig. 5b) or antibody staining for Echinoid we observed expression of both in glial cells and NSCs during quiescence (Fig. 6a,b and Supplementary Fig. 5a-c). Since our data so far suggests a cell-contact inhibition of growth by niche glial cells we analysed whether Crumbs::GFP localizes to contact sites of glial cells and NSCs. Indeed, we could observe a slight accumulation in NSCs towards the contact site with glial cells
(Supplementary Fig. 5d). To prove that NSC and glial cells indeed form cell-cell contacts, we stained for E-Cadherin and could monitor adherens junctions between glial cells and NSCs (Supplementary Fig. 5e). Crumbs::GFP expression in glial cells and NSCs was lost over time (8 and $24 \mathrm{~h} \mathrm{ALH}$, respectively) whereas Echinoid was downregulated mainly in glial cells (24 h, Fig. 6a,b and Supplementary Fig. 5a,c). Nutritional deprivation prolongs quiescence and Crumbs::GFP, $c r b$-mRNA or Echinoid expression was maintained in glial cells and NSCs at $24 \mathrm{~h} \mathrm{ALH} \mathrm{(Fig.} \mathrm{6a,b} \mathrm{and} \mathrm{Supplementary} \mathrm{Fig.} \mathrm{5a,c,f),}$ whereas early activation of the Insulin-like receptor signalling leads to premature loss of Crumbs::GFP in NSCs (Supplementary Fig. 5g). Thus, Crumbs and Echinoid are expressed in nonepithelial niche glial cells and NSCs during the phase of quiescence and are developmentally downregulated in response to nutrition.

Ectopic $c r b$ causes decreased NSC growth and proliferation. Next we assessed whether ectopic expression of $c r b$ or $e d$ would prolong quiescence. Expression of $c r b$ in glial cells leads to embryonic lethality, and its expression in brain NSCs results in cell clustering and an increase in NSCs of the MBNB (Supplementary Fig. 5h) complicating the analysis. These phenotypes were not apparent in NSCs of the VNC and we were able to analyse their growth behaviour and their 

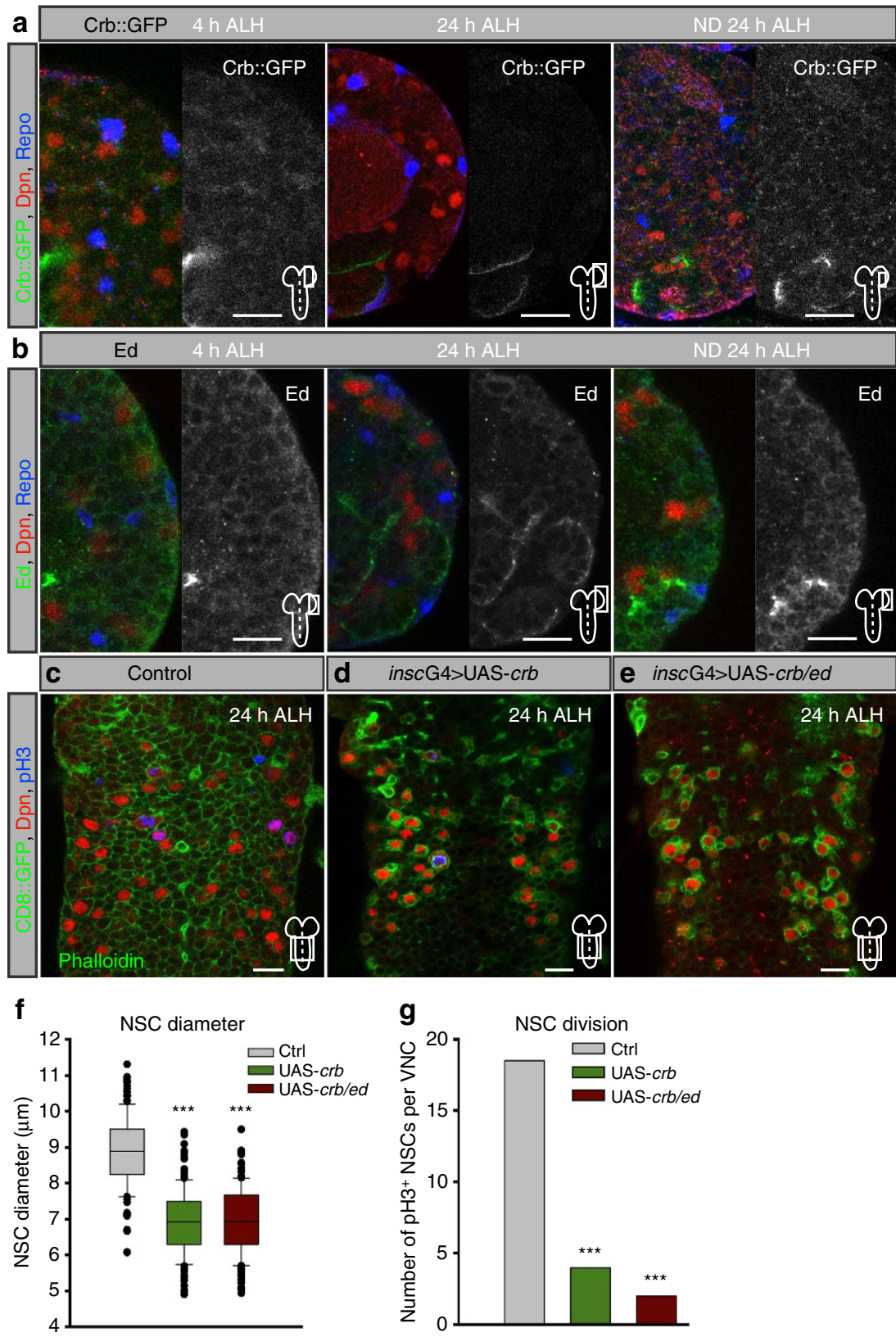

Figure 6 | Crumbs and Echinoid are temporally expressed in glial cells and NSCs during quiescence and prolonged expression suppresses NSCs growth and proliferation. (a) Expression of Crumbs::GFP at 4, 24 and $24 \mathrm{~h}$ in nutrition-deprived (ND) ALH. Right side of each panel shows the GFP channel in monochrome. Crumbs expression is lost in both glial and NSCs after reactivation but persists in ND. (b) Expression of Echinoid at 4, 24 and $24 \mathrm{~h}$ in nutrition-deprived (ND) ALH. Right side of each panel shows the Echinoid channel in monochrome. Echinoid expression is lost in glial cells at $24 \mathrm{~h}$ ALH, while NSCs still express Echinoid, but persists in ND. (c-e) In comparison with wild type (c) prolonged expression in NSCs of crb (d) or crb/ed (e) leads to suppression of NSCs cell growth and division in ventral nerve cord NSCs at $24 \mathrm{~h} \mathrm{ALH}$. (f, $\mathbf{g}$ ) Quantification of NSC cell diameters and mitosis upon NSCspecific prolonged expression of $c r b$ or $\mathrm{crb} / \mathrm{ed}$ at $24 \mathrm{~h} \mathrm{ALH}$. (f) ${ }^{\star \star \star} P<0.001$. Wilcoxon rank sum test. Median and s.d. were calculated from two biological replicates. Control VNCs $n=129$ NSCs (3 VNCs); inscG4 > UAS-crb, $n=144$ NSCs (3 VNCs); inscG4>UAS-crb/ed, $n=165$ NSCs (3 VNCs). (g) Ratio of NSCs in mitosis (pH3-positive) per counted VNCs at $24 \mathrm{~h} \mathrm{ALH}$ in wild type of $3 \mathrm{VNC}$ and inscG4 > UAS-crb of 3 VNCs and inscG $4>$ UAS-crb/ed of 3 VNCs. All images are single focal planes, anterior up. Scale bar, $10 \mu \mathrm{m}$. See also Supplementary Fig. 5.

mitotic index. Expression of crb was not able to suppress reactivation, but was sufficient to strongly reduce growth and proliferation at $24 \mathrm{~h} \mathrm{ALH} \mathrm{(Fig.} 6 \mathrm{c}-\mathrm{g}$ ). Co-expression of $\mathrm{crb}$ and ed had no additive effect on growth suppression, but the mitotic index was further reduced (Fig. 6e-g). Thus, although we observed a strong reduction in growth and proliferation, ectopic crb and ed did not extend quiescence, which might be owing to the nutritional reactivatory signal overcoming $c r b$ input.
SHW discriminates between quiescent and non-quiescent NSCs. Next we sought to investigate whether the SHW discriminates quiescent from non-quiescent NSCs in Drosophila. Analysing the growth behaviour and mitotic index of non-quiescent NSCs of the mushroom bodies (MBNBs) at 4, 24 and $48 \mathrm{~h} \mathrm{ALH}$ and in nutritional deprivation unravelled that their growth and proliferation depends on nutrition and requires active regulation (Fig. 7a,b). Testing the SHW we observed constant nuclear Yorkie levels in MBNBs (Fig. 7c) and expression of Fj-lacZ, expanded and activity 
of bantam (Fig. 4a and Supplementary Fig. 3a,b) confirming a continuous Yorkie activity. Indeed, in $y k i^{B 5}$ mutants we could observe a lack of MBNBs growth and a marked reduction in the proliferative capacity of MBNBs at $48 \mathrm{~h}$ ALH (Fig. 7a,b,d) compared with wild-type MBNBs (Fig. 7e). We conclude that continuous Yorkie activity is necessary for MBNBs growth and proliferation and Yorkie activity discriminates between quiescent and non-quiescent NSCs in Drosophila.

\section{Discussion}

NSCs need to tightly control the balance between proliferation and quiescence, since deregulation can lead to tumour formation
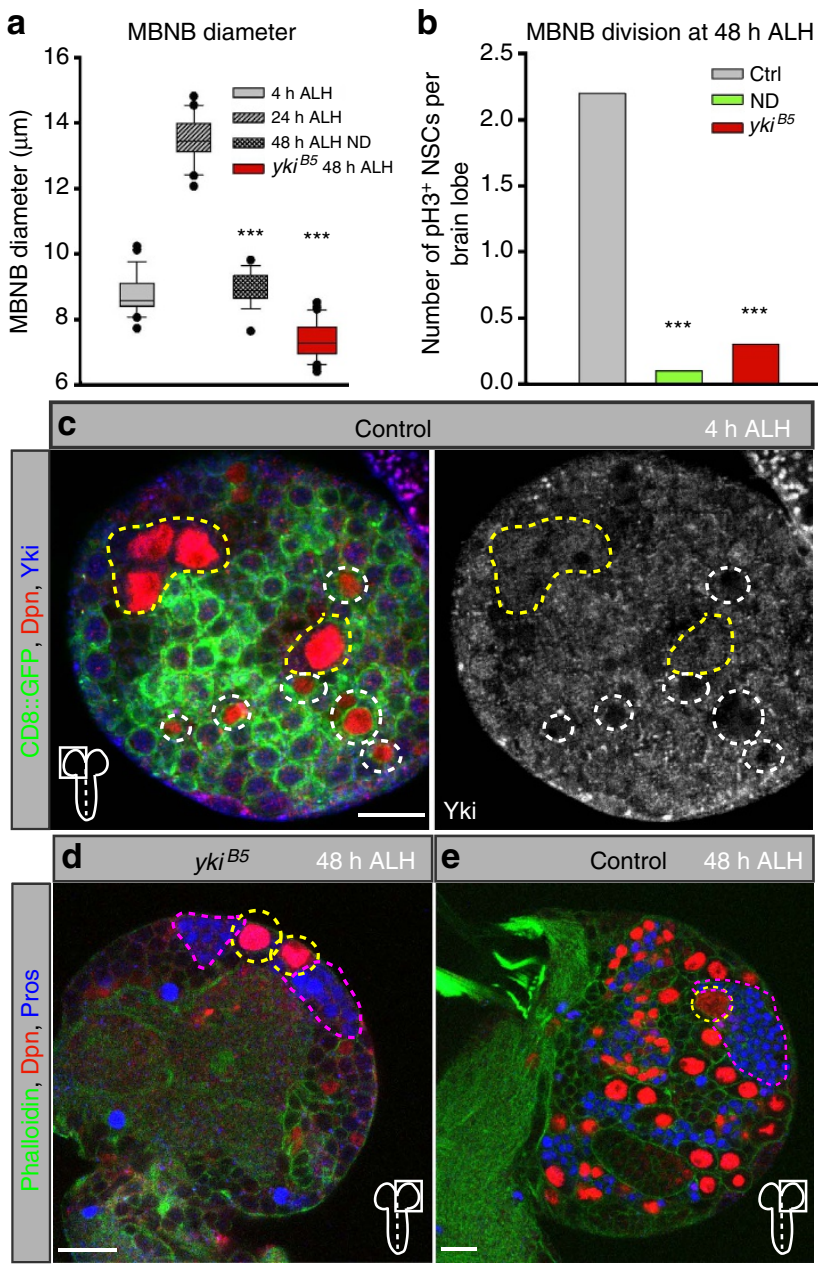

f Developmental timing
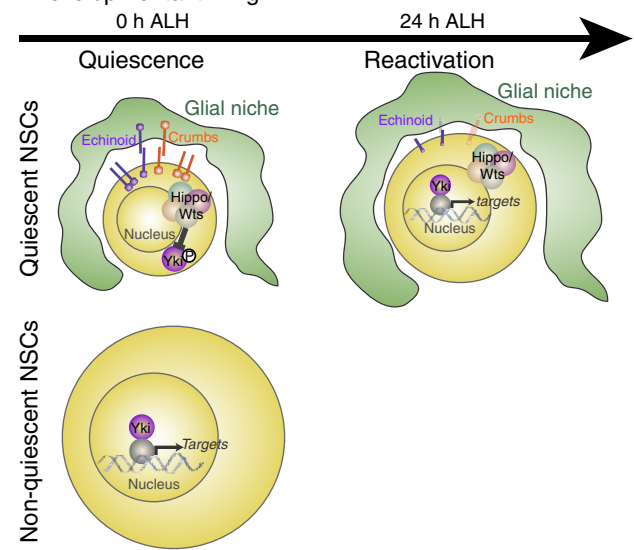

or premature depletion of the progenitor pool ${ }^{1,3}$. In order to orchestrate their behaviour according to the status of the organism they need to communicate with their surrounding microenvironment. Similar to the neurogenic niche in vertebrates $^{49}$, processes of glial cells in insects enwrap NSCs to form an enclosed chamber known as the trophospongium ${ }^{50}$. It is still debated whether Drosophila NSCs are independent of niche signalling and indeed NSCs in culture undergo asymmetric cell division to self-renew and produce differentiating progeny ${ }^{51,52}$, exhibiting very similar behaviour as their counterparts in vivo, like progressing through an intrinsically regulated series of transcription factors (temporal transcription factor cascade) $)^{53}$ and generating diverse cell types and lineages in vitro resembling the in vivo lineages in cell number and identity ${ }^{54}$. Conversely, loss of contact to the surrounding epithelium leads to a randomization of the mitotic spindle in isolated embryonic $\mathrm{NSCs}^{55}$ and compromising Drosophila E-Cadherin function in niche glial cells impairs the mitotic activity of $\mathrm{NSCs}^{56}$. During quiescence and reactivation NSCs show a strong dependency on extrinsic signals from glial cells. Glial cells express and secrete quiescence promoting factors ${ }^{13}$, or in response to nutrition, activating factors ${ }^{10,11}$. We now show that niche glial cells express transmembrane proteins Crumbs and Echinoid that act in trans to activate the SHW in NSCs to maintain quiescence and suppress cell growth. Thus, like vertebrate adult NSCs, Drosophila NSCs show different degrees of dependency on niche signalling. During quiescence both NSC populations need extrinsic cues from the niche to maintain quiescence (for example, this work $^{10,11,57,58}$, whereas during the active-phase lineage progression maybe more cell-intrinsic, pre-programed and to a less degree depending on the niche $e^{54,59}$.

The SHW and its effector Yorkie/YAP have been widely implicated in stem cell biology and organ size restriction. In the vertebrate liver progenitors, the hepatocytes, the SHW controls quiescence of these stem cells ${ }^{60}$. Combined loss of Mst1/2 (homologues of Drosophila Hippo) resulted in loss of YAP phosphorylation, leading to a massive overgrowth and hepatocellular carcinoma. Other regenerative tissues, like the skin or the intestine, also harbour stem cells and an involvement of the SHW was likewise shown ${ }^{61-65}$. Our data now show that the SHW is also important for the regulation of quiescence in NSCs. The SHW is active during quiescence and suppresses

\section{Figure 7 | Yorkie activity discriminates between quiescent and} non-quiescent NSCs. (a) Quantification of the cell diameter of NSCs of the mushroom bodies (MBNB) at 4, 24, $48 \mathrm{~h}$ in nutrition-deprived (ND) or $y k^{B 5}$ mutants at $48 \mathrm{~h} \mathrm{ALH}$. ${ }^{\star \star \star} P<0.001$. Wilcoxon rank sum test. Median and s.d. were calculated from two biological replicates. Control at $4 \mathrm{~h} n=28$ NSCs ( 7 brain lobes); $24 \mathrm{~h} n=20$ NSCs ( 5 brain lobes); $48 \mathrm{~h} \mathrm{ND} n=19$ NSCs ( 5 brain lobes); $y k i^{B 5}$ at $48 \mathrm{~h} n=40$ NSCs (10 brain lobes).

(b) Quantification of MBNB in mitosis. Number of pH3-positive NSCs per brain lobes counted in 7 control brain lobes, 5 brain lobes in ND and 10 brain lobes in $y k^{B 5}$ mutants. (c) Yorkie is constantly nuclear in nonquiescent NSCs (yellow circles) and not nuclear in quiescent NSCs (cyan circles) at $4 \mathrm{~h} \mathrm{ALH}$. (d,e) NSCs of the MBNB in $y k^{B 5}$ (d) are smaller and have less Prospero-positive (Pros, blue encircled in magenta) progeny than compared with the control (e) at $48 \mathrm{~h} \mathrm{ALH}$. (f) Schematic of Crumbsand Echinoid-mediated cell-contact inhibition of growth through the Hippo signalling pathway (upper panel). Homophilic interaction of Crumbs and Echinoid in trans on the glial niche and on the NSC inhibits Yorkie. During reactivation Crumbs and Echinoid are downregulated in response to nutrition in the niche, which inactivates the Hippo pathway and activates Yorkie. Yorkie is necessary and sufficient for reactivation, growth and proliferation. Non-quiescent NSCs have constant Yorkie activity (lower panel). All images are single focal planes, anterior up. Scale bar, $10 \mu \mathrm{m}$. 
inappropriate growth and proliferation of NSC in the Drosophila larvae. Similar to Drosophila, mouse adult quiescent NSCs (aNSCs) also show a prominent cell growth before the initiation of proliferation 59,66 . Thus, a similar mechanism of promoting quiescence by growth restriction might exist in adult vertebrate NSCs. Indeed, a recent molecular study on NSC quiescence showed that multiple SHW members like Lats2 (Warts homologue) or WWC2 (Kibra homologue) are upregulated in aNSCs, which after BMP4 exposure enter into a quiescence-like status in cell culture ${ }^{57}$. Moreover, our study demonstrates that a crosstalk between the niche glial cells and the stem cells via Crumbs and Echinoid activates the SHW to repress growth during quiescence. This might also be conserved in mouse aNSCs since Martynoga et al. ${ }^{57}$ showed that upon BMP4-induced quiescence expression of crumbs2 (CRB2, Drosophila crumbs homologue) is also upregulated in aNSCs. Whether CRB2 is expressed in the vertebrate niche and activates the SHW in NSCs during quiescence still has to be shown. Nevertheless, given these similarities it is attractive to speculate that the Hippo pathway might act as a general regulator of NSC quiescence in vertebrates and invertebrates.

In Drosophila two different populations of NSCs can be discriminated: NSCs that become significantly smaller and enter quiescence at the end of embryonic stages, and NSCs that constantly proliferate and do not go into quiescence. How this difference is established is not known up to now. Here we show that the activity of the transcriptional regulator Yorkie seems to be a major difference between these two populations of NSCs. Although quiescent NSCs have active Hippo signalling and thus no active Yorkie, the non-quiescent NSCs show constant nuclear Yorkie and constant expression of the known Yorkie-targets bantam and Four-jointed. We did not observe failure to enter into quiescence upon wts-RNAi or in trans-heterozygous hpo mutants and thus it seems less likely that SHW activation is needed to initiate quiescence.

Non-quiescent NSCs increase in size upon larval hatching and like in quiescent NSCs, this growth depends on nutrition and on Yorkie function. This also influences the proliferative capacity, since both populations of NSCs either showed no proliferation or exited proliferation prematurely. A similar function of YAP was described during vertebrate neurogenesis in the developing chick neural tube. YAP is highly expressed in NSCs and co-localizes with Sox2 a neural progenitor marker. Loss of YAP leads to premature differentiation, whereas overexpression leads to an increase in the progenitor pool and accelerated cell cycle progression ${ }^{67}$. More recently YAP expression was also found in the mouse ventricular zone in Sox2-positive progenitors ${ }^{68}$ and, importantly, simultaneous depletion of YAP and FAT4 or Dachs 1 rescued a prolonged neuroprogenitor cell proliferation phenotype in a mouse model for Van Maldergem syndrome ${ }^{69}$. Therefore, Yorkie/YAP emerges as an important regulator of NSC biology and it is therefore of great importance to identify the precise molecular mechanisms and target genes by which Yorkie/YAP promote growth, proliferation and stem cell identity in NSCs. Interestingly, in Drosophila we could uncover an essential difference between NSCs in the brain and the VNC. In both populations of stem cells Yorkie activates its well-established target bantam. Yet, loss of bantam severely impaired growth and proliferation of the VNC NSCs, whereas the effects were milder in brain NSCs. Thus, to fully understand the function of Yorkie in NSCs it will be important in the future to unravel the stem cell-specific target genes that are regulated by Yorkie in NSCs.

\section{Methods}

Genetics. The RNAi fly strains obtained from the Vienna Drosophila RNAi Center (VDRC) are: expanded-RNAi (Stock number: 22994), kibra-RNAi (100765),
Merlin-RNAi (7161), crumbs-RNAi (39177), echinoid-RNAi (104279), hippo-RNAi (104196), warts-RNAi (106174). The RNAi fly strains obtained from the Bloomington Drosophila Stock Center (BSC) are: hippo-RNAi (Stock number: 33614), warts-RNAi (34064), crumbs-RNAi (38373), 14-3-3zeta-RNAi (31498). NSC-specific RNAi was performed with insc-GAL4 (w ${ }^{1118}$; $\mathrm{P}\{\mathrm{GawB}\}$ inscMZ1407), glial-specific RNAi was performed with repo-GAL4 (w ${ }^{1118}$; P\{GAL4\}repo/TM6b, iab-lacZ). Both GAL4 driver lines carried UAS-CD8::gfp or UAS-CD4td::gfp and UAS-dicer2. For larval-restricted RNAi we combined the insc-GAL4, UAS-CD8::gfp with the tubP-GAL80[ts] (Bloomington Stock 7108) and embryonic phases were cultured at $18^{\circ} \mathrm{C}$ before shifting to $29^{\circ} \mathrm{C}$ just ALH. All other RNAi experiments were conducted at $29^{\circ} \mathrm{C}$ during larval life-embryonic phases were cultured at $25^{\circ} \mathrm{C}$. Mutants alleles used were, $e d^{F 72}, h p o^{J M 1}, h p o^{K C 202}, y k i^{B 5}$ and band ${ }^{1}$ balanced over $C y O, P w[+\mathrm{mC}]=$ Dfd-EYFP2, $C y O, \mathrm{P}(\mathrm{GAL} 4-t w i . \mathrm{G}) 2.2$, $\mathrm{P}(\mathrm{UAS}-2 \mathrm{xEGFP}) \mathrm{AH} 2.2$ or TM6B, $\mathrm{Pw}[+\mathrm{mC}]=\mathrm{Dfd}-\mathrm{EYFP} 3, S b^{1} \mathrm{~Tb}^{1} c a^{1}$ UAS- $y k i^{\text {S168A }}$ (Stock number: 28818), UAS-crb (5544) and fj-LacZ (6370) were obtained from the BSC. The fly stock carrying the crumbs-extracellular domain tagged with GFP (Crumbs::GFP-A/TM3) was a kind gift from Yang Hong ${ }^{48}$. For nutritional deprivation egg collection were made on and hatched larvae transferred to agar plates prepared with $11 \mathrm{PBS}, 10 \mathrm{~g}$ agar, $50 \mathrm{~g}$ sucrose. For well-fed larvae collection, flies were reared on standard Drosophila fly food and egg collections were made on and hatched larvae transferred to agar plates prepared with 11 apple juice, $27 \mathrm{~g}$ agar supplemented with dry yeast.

Antibodies, in situ probes and immunohistochemistry. Immunohistochemistry experiments were performed as previously described ${ }^{70}$. In brief, larval CNSs were dissected in PBS, fixed in $4 \%$ formaldehyde. Washing steps were performed using PBS with $0.3 \%$ Triton X-100 $(3 \times 5 \mathrm{~min})$ and primary antibodies were incubated overnight at $4{ }^{\circ} \mathrm{C}$. Antibodies used were guinea pig anti-Deadpan $(1: 1,000$, kind gift from Jürgen Knoblich), mouse anti-Pros (1:100, MR1A, Developmental Studies Hybridoma Bank, DSHB), mouse anti-Repo (1:100, 8D12, DSHB), mouse anti-Dlg (1:20, DSHB), mouse anti-pH3 (1:1,000, Cell Signaling Technology), mouse anti- $\beta$-Gal (1:375, Promega), mouse anti-Dig (1:1,000, Roche), antiEchinoid (1:1,000, kind gift from Laura Nilson), rabbit anti-Yorkie (1:400, kind gift from Kenneth Irvine), rabbit anti-Expanded (1:1,000, kind gift from Richard Fehon).

In situ probe for crumbs was PCR-generated using the following forward primers: $5^{\prime}$-CGTTGGTGGCCAGAAATTGG- $3^{\prime}$ and $5^{\prime}$-CACAGTGCTGACCCT CGAAT- $3^{\prime}$ (5'-TAATACGACTCACTATAGGAGACCAC- $\left.3^{\prime}\right)$ as reverse primer $((\mathrm{XX})=\mathrm{T} 7$ sequence $)$. After purification of the PCR product the in vitro transcription using T7 and the Dig RNA Labelling Mix (Roche) was used to generate the RNA in situ probe.

EdU incorporation assay. To detect mitotic activity using EdU incorporation we dissected larval CNS at appropriate time points and incubated them for $1 \mathrm{~h}$ in $10 \mathrm{mM}$ EdU/PBS. CNSs were fixed for $15 \mathrm{~min}$ in $4 \%$ formaldehyde/PBS and Alexa Fluor azide was detected according to the manufacturer's instructions (Click-iT EdU Imaging Kit, Invitrogen).

Image acquisition and processing. Confocal images were acquired on a Leica SP5 confocal laser-scanning microscope using $\times 63$ glycine immersion objective lens or a Leica SP8 confocal laser-scanning microscope using $\times 40$ objective lens. All images represent single confocal sections except for images of EdU incorporation in $e d^{F 72}$ mutants that are projections of $\mathrm{z}$-stacks. Images were processed using the Leica LAS, Adobe Photoshop and assembled in Adobe Illustrator. Cell size measurements and $\mathrm{pH} 3$-cell counts were done using the Leica LAS, statistical analysis were conducted using SigmaPlot. The medial cell body of NSCs were measured for cell diameter evaluation excluding the longest and the shortest axis of the cell.

Intensity measurements for Yorkie localization. The Leica LAS AF software was used to determine the pixel intensities of each channel across a cell. The values for each channel were exported to Excel and the whole cell and nuclear areas were determined by the signal intensity of the Deadpan staining (nucleus) and the Phalloidin staining (outer cell membrane). The intersection was defined as cytoplasm. According to this definition the Yorkie signal intensity values were separated into nuclear and cytoplasmic fraction and pixel intensities were averaged for each fraction. The ratio between the means was calculated as a measure for the relative amount of protein per sub-compartment. SigmaPlot was used for statistical analysis.

\section{References}

1. Knoblich, J. A. Asymmetric cell division: recent developments and their implications for tumour biology. Nat. Rev. Mol. Cell Biol. 11, 849-860 (2010)

2. Homem, C. C. \& Knoblich, J. A. Drosophila neuroblasts: a model for stem cell biology. Development 139, 4297-4310 (2012)

3. Cheung, T. H. \& Rando, T. A. Molecular regulation of stem cell quiescence. Nat. Rev. Mol. Cell Biol. 14, 329-340 (2013).

4. Coller, H. A., Sang, L. \& Roberts, J. M. A new description of cellular quiescence. PLoS Biol. 4, e83 (2006). 
5. Prokop, A. \& Technau, G. M. The origin of postembryonic neuroblasts in the ventral nerve cord of Drosophila melanogaster. Development 111, 79-88 (1991).

6. Tsuji, T., Hasegawa, E. \& Isshiki, T. Neuroblast entry into quiescence is regulated intrinsically by the combined action of spatial Hox proteins and temporal identity factors. Development 135, 3859-3869 (2008).

7. Lai, S. L. \& Doe, C. Q. Transient nuclear Prospero induces neural progenitor quiescence. Elife 3, e03363 (2014).

8. Britton, J. S. \& Edgar, B. A. Environmental control of the cell cycle in Drosophila: nutrition activates mitotic and endoreplicative cells by distinct mechanisms. Development 125, 2149-2158 (1998).

9. Colombani, J. et al. A nutrient sensor mechanism controls Drosophila growth. Cell 114, 739-749 (2003).

10. Chell, J. M. \& Brand, A. H. Nutrition-responsive glia control exit of neural stem cells from quiescence. Cell 143, 1161-1173 (2010).

11. Sousa-Nunes, R., Yee, L. L. \& Gould, A. P. Fat cells reactivate quiescent neuroblasts via TOR and glial insulin relays in Drosophila. Nature 471, 508-512 (2011).

12. Truman, J. W. \& Bate, M. Spatial and temporal patterns of neurogenesis in the central nervous system of Drosophila melanogaster. Dev. Biol. 125, 145-157 (1988).

13. Ebens, A. J., Garren, H., Cheyette, B. N. \& Zipursky, S. L. The Drosophila anachronism locus: a glycoprotein secreted by glia inhibits neuroblast proliferation. Cell 74, 15-27 (1993).

14. Halder, G. \& Johnson, R. L. Hippo signaling: growth control and beyond. Development 138, 9-22 (2011).

15. Harvey, K. \& Tapon, N. The Salvador-Warts-Hippo pathway-an emerging tumour-suppressor network. Nat. Rev. Cancer 7, 182-191 (2007).

16. Pan, D. The hippo signaling pathway in development and cancer. Dev. Cell 19, 491-505 (2010).

17. Oh, H. \& Irvine, K. D. In vivo regulation of Yorkie phosphorylation and localization. Development 135, 1081-1088 (2008).

18. Dong, J. et al. Elucidation of a universal size-control mechanism in Drosophila and mammals. Cell 130, 1120-1133 (2007).

19. Wu, S., Liu, Y., Zheng, Y., Dong, J. \& Pan, D. The TEAD/TEF family protein Scalloped mediates transcriptional output of the Hippo growth-regulatory pathway. Dev. Cell 14, 388-398 (2008).

20. Zhang, L. et al. The TEAD/TEF family of transcription factor Scalloped mediates Hippo signaling in organ size control. Dev. Cell 14, 377-387 (2008).

21. Pan, D. Hippo signaling in organ size control. Genes Dev. 21, 886-897 (2007).

22. Saucedo, L. J. \& Edgar, B. A. Filling out the Hippo pathway. Nat. Rev. Mol. Cell Biol. 8, 613-621 (2007).

23. Yu, F. X. \& Guan, K. L. The Hippo pathway: regulators and regulations. Genes Dev. 27, 355-371 (2013).

24. Tremblay, A. M. \& Camargo, F. D. Hippo signaling in mammalian stem cells. Semin. Cell Dev. Biol. 23, 818-826 (2012).

25. Reddy, B. V., Rauskolb, C. \& Irvine, K. D. Influence of Fat-Hippo and Notch signaling on the proliferation and differentiation of Drosophila optic neuroepithelia. Development 137, 2397-2408 (2010).

26. Richter, C., Oktaba, K., Steinmann, J., Muller, J. \& Knoblich, J. A. The tumour suppressor L(3)mbt inhibits neuroepithelial proliferation and acts on insulator elements. Nat. Cell Biol. 13, 1029-1039 (2011)

27. Reddy, B. V. \& Irvine, K. D. Regulation of Drosophila glial cell proliferation by Merlin-Hippo signaling. Development 138, 5201-5212 (2011).

28. Neumuller, R. A. et al. Genome-wide analysis of self-renewal in Drosophila neural stem cells by transgenic RNAi. Cell Stem Cell 8, 580-593 (2011).

29. Ito, K. \& Hotta, Y. Proliferation pattern of postembryonic neuroblasts in the brain of Drosophila melanogaster. Dev. Biol. 149, 134-148 (1992).

30. Jia, J., Zhang, W., Wang, B., Trinko, R. \& Jiang, J. The Drosophila Ste20 family kinase dMST functions as a tumor suppressor by restricting cell proliferation and promoting apoptosis. Genes Dev. 17, 2514-2519 (2003).

31. Udan, R. S., Kango-Singh, M., Nolo, R., Tao, C. \& Halder, G. Hippo promotes proliferation arrest and apoptosis in the Salvador/Warts pathway. Nat. Cell Biol. 5, 914-920 (2003)

32. Huang, J., Wu, S., Barrera, J., Matthews, K. \& Pan, D. The Hippo signaling pathway coordinately regulates cell proliferation and apoptosis by inactivating Yorkie, the Drosophila homolog of YAP. Cell 122, 421-434 (2005).

33. Cho, E. et al. Delineation of a Fat tumor suppressor pathway. Nat. Genet. 38, 1142-1150 (2006).

34. Hamaratoglu, F. et al. The tumour-suppressor genes NF2/Merlin and Expanded act through Hippo signalling to regulate cell proliferation and apoptosis. Nat. Cell Biol. 8, 27-36 (2006).

35. Nolo, R., Morrison, C. M., Tao, C., Zhang, X. \& Halder, G. The bantam MicroRNA is a target of the hippo tumor-suppressor pathway. Curr. Biol. 16, R853-R855 (2006)
36. Brennecke, J., Hipfner, D. R., Stark, A., Russell, R. B. \& Cohen, S. M. bantam encodes a developmentally regulated microRNA that controls cell proliferation and regulates the proapoptotic gene hid in Drosophila. Cell 113, 25-36 (2003)

37. Hipfner, D. R., Weigmann, K. \& Cohen, S. M. The bantam gene regulates Drosophila growth. Genetics 161, 1527-1537 (2002).

38. Thompson, B. J. \& Cohen, S. M. The Hippo pathway regulates the bantam microRNA to control cell proliferation and apoptosis in Drosophila. Cell 126, 767-774 (2006).

39. Robinson, B. S., Huang, J., Hong, Y. \& Moberg, K. H. Crumbs regulates Salvador/Warts/Hippo signaling in Drosophila via the FERM-domain protein Expanded. Curr. Biol. 20, 582-590 (2010).

40. Grzeschik, N. A., Parsons, L. M., Allott, M. L., Harvey, K. F. \& Richardson, H. E. $\mathrm{Lgl}$, aPKC, and Crumbs regulate the Salvador/Warts/Hippo pathway through two distinct mechanisms. Curr. Biol. 20, 573-581 (2010).

41. Ling, C. et al. The apical transmembrane protein Crumbs functions as a tumor suppressor that regulates Hippo signaling by binding to Expanded. Proc. Natl Acad. Sci. USA 107, 10532-10537 (2010).

42. Chen, C. L. et al. The apical-basal cell polarity determinant Crumbs regulates Hippo signaling in Drosophila. Proc. Natl Acad. Sci. USA 107, 15810-15815 (2010).

43. Chang, L. H. et al. Differential adhesion and actomyosin cable collaborate to drive Echinoid-mediated cell sorting. Development 138, 3803-3812 (2011).

44. Yue, T., Tian, A. \& Jiang, J. The cell adhesion molecule echinoid functions as a tumor suppressor and upstream regulator of the Hippo signaling pathway. Dev. Cell 22, 255-267 (2012).

45. Letizia, A., Ricardo, S., Moussian, B., Martin, N. \& Llimargas, M. A functional role of the extracellular domain of Crumbs in cell architecture and apicobasal polarity. J. Cell Sci. 126, 2157-2163 (2013).

46. Tepass, U., Theres, C. \& Knust, E. crumbs encodes an EGF-like protein expressed on apical membranes of Drosophila epithelial cells and required for organization of epithelia. Cell 61, 787-799 (1990).

47. Laplante, C. \& Nilson, L. A. Differential expression of the adhesion molecule Echinoid drives epithelial morphogenesis in Drosophila. Development 133, 3255-3264 (2006).

48. Huang, J., Zhou, W., Dong, W., Watson, A. M. \& Hong, Y. Directed, efficient, and versatile modifications of the Drosophila genome by genomic engineering. Proc. Natl Acad. Sci. USA 106, 8284-8289 (2009).

49. Bjornsson, C. S., Apostolopoulou, M., Tian, Y. \& Temple, S. It takes a village: constructing the neurogenic niche. Dev. Cell 32, 435-446 (2015).

50. Hoyle, G. Glial cells of an insect ganglion. J. Comp. Neurol. 246, 85-103 (1986).

51. Ceron, J., Tejedor, F. J. \& Moya, F. A primary cell culture of Drosophila postembryonic larval neuroblasts to study cell cycle and asymmetric division. Eur. J. Cell Biol. 85, 567-575 (2006).

52. Homem, C. C., Reichardt, I., Berger, C., Lendl, T. \& Knoblich, J. A. Long-term live cell imaging and automated $4 \mathrm{D}$ analysis of neuroblast lineages. PLOS ONE 8, e79588 (2013).

53. Brody, T. \& Odenwald, W. F. Programmed transformations in neuroblast gene expression during Drosophila CNS lineage development. Dev. Biol. 226, 34-44 (2000).

54. Lüer, K. \& Technau, G. M. Single cell cultures of Drosophila neuroectodermal and mesectodermal central nervous system progenitors reveal different degrees of developmental autonomy. Neural. Dev. 4, 30 (2009).

55. Siegrist, S. E. \& Doe, C. Q. Extrinsic cues orient the cell division axis in Drosophila embryonic neuroblasts. Development 133, 529-536 (2006).

56. Dumstrei, K., Wang, F. \& Hartenstein, V. Role of DE-cadherin in neuroblast proliferation, neural morphogenesis, and axon tract formation in Drosophila larval brain development. J. Neurosci. 23, 3325-3335 (2003).

57. Martynoga, B. et al. Epigenomic enhancer annotation reveals a key role for NFIX in neural stem cell quiescence. Genes Dev. 27, 1769-1786 (2013).

58. Mira, H. et al. Signaling through BMPR-IA regulates quiescence and long-term activity of neural stem cells in the adult hippocampus. Cell Stem Cell 7, 78-89 (2010).

59. Costa, M. R. et al. Continuous live imaging of adult neural stem cell division and lineage progression in vitro. Development 138, 1057-1068 (2011).

60. Zhou, D. et al. Mst1 and Mst2 maintain hepatocyte quiescence and suppress hepatocellular carcinoma development through inactivation of the Yap1 oncogene. Cancer Cell 16, 425-438 (2009).

61. Schlegelmilch, K. et al. Yap1 acts downstream of alpha-catenin to control epidermal proliferation. Cell 144, 782-795 (2011).

62. Zhang, H., Pasolli, H. A. \& Fuchs, E. Yes-associated protein (YAP) transcriptional coactivator functions in balancing growth and differentiation in skin. Proc. Natl Acad. Sci. USA 108, 2270-2275 (2011).

63. Barry, E. R. et al. Restriction of intestinal stem cell expansion and the regenerative response by YAP. Nature 493, 106-110 (2013).

64. Zhou, D. et al. Mst1 and Mst2 protein kinases restrain intestinal stem cell proliferation and colonic tumorigenesis by inhibition of Yes-associated protein (Yap) overabundance. Proc. Natl Acad. Sci. USA 108, E1312-E1320 (2011). 
65. Staley, B. K. \& Irvine, K. D. Warts and Yorkie mediate intestinal regeneration by influencing stem cell proliferation. Curr. Biol. 20, 1580-1587 (2010).

66. Ortega, F. et al. Using an adherent cell culture of the mouse subependymal zone to study the behavior of adult neural stem cells on a single-cell level. Nat. Protoc. 6, 1847-1859 (2011).

67. Cao, X., Pfaff, S. L. \& Gage, F. H. YAP regulates neural progenitor cell number via the TEA domain transcription factor. Genes Dev. 22, 3320-3334 (2008).

68. Li, Y., Hibbs, M. A., Gard, A. L., Shylo, N. A. \& Yun, K. Genome-wide analysis of N1ICD/RBPJ targets in vivo reveals direct transcriptional regulation of Wnt, SHH, and hippo pathway effectors by Notch1. Stem Cells 30, 741-752 (2012).

69. Cappello, S. et al. Mutations in genes encoding the cadherin receptor-ligand pair DCHS1 and FAT4 disrupt cerebral cortical development. Nat. Genet. 45, 1300-1308 (2013).

70. Berger, C. et al. FACS purification and transcriptome analysis of Drosophila neural stem cells reveals a role for Klumpfuss in self-renewal. Cell Rep. 2, 407-418 (2012).

\section{Acknowledgements}

We thank the Vienna Drosophila RNAi Centre and the Bloomington Drosophila stock Center for their services. We are grateful to Georg Halder, Barry Thompson, Julius Brennicke, Nicolas Tapon, Kenneth Irvine, Richard Fehon, Jürgen Knoblich, Jui-Chou Hsu, Yang Hong, Jin Jiang and Laura Nilson for stocks and antibodies. We thank Nicolas Tapon and Ieva Gailite for discussing data before publication and comments on the manuscript. R.D. is supported by a Rhineland-Palatine fellowship. C.S.B. was supported by a Leverhulme Trust UK Early Career Fellowship (ECF/2010/0526) and Plymouth Peninsula School of Medicine. T.B. was supported by Plymouth School of Biomedical and Healthcare Sciences. The Berger laboratory is supported by the DFG (BE4278/1-1, BE4278/3-1) and the University of Mainz.

\section{Author contributions}

All the authors designed the experiments. R.D., K.W., C.S.B. and T.B. performed the experiments. R.D. and C.B. wrote the manuscript.

\section{Additional information}

Supplementary Information accompanies this paper at http://www.nature.com/ naturecommunications

Competing financial interests: The authors declare no competing financial interests.

Reprints and permission information is available online at http://npg.nature.com/ reprintsandpermissions/

How to cite this article: Ding, R. et al. The Hippo signalling pathway maintains quiescence in Drosophila neural stem cells. Nat. Commun. 7:10510 doi: 10.1038/ ncomms10510 (2016).

\section{(c) (i)}

This work is licensed under a Creative Commons Attribution 4.0 International License. The images or other third party material in this article are included in the article's Creative Commons license, unless indicated otherwise in the credit line; if the material is not included under the Creative Commons license, users will need to obtain permission from the license holder to reproduce the material. To view a copy of this license, visit http://creativecommons.org/licenses/by/4.0/ 